\title{
Algorithm for Improving the Restorability of Power Supply in Distribution Systems
}

\author{
Youman Deng, Senior Member, IEEE, Le Cai, and Yixin Ni, Senior Member, IEEE
}

\begin{abstract}
In this paper, a new concept of restorability of power supply (RPS) and the corresponding performance indices are suggested. Load transfer algorithm is used for enhancing the RPS of distribution systems. Feeder incidence diagram (FID) is proposed for analyzing the rules for load transfer. The performance indices, maximal interrupted power (MIP), and maximal number of interrupted customers (MNIC), will be calculated before the restoration operation or apparatus maintenance. These indices can serve for evaluating the operation status of feeders, estimating the impact of load transfer and switch operation, and assessing the RPS. They can also be used to optimize the allocation of tie-breakers in a meshed network. The work is of great significance in distribution system reconstruction in China.
\end{abstract}

Index Terms-Distribution systems, load transfer, restorability of power supply.

\section{NOMENCLATURE}

RPS Restorability of power supply.

MIP Maximal interrupted power $(\mathrm{kW})$.

$M N I C \quad$ Maximal number of interrupted customers.

FID Feeder incidence diagram.

$R_{M I P} \quad$ Maximum rate of interrupted power.

$P_{D i} \quad$ Total active load of feeder $i(\mathrm{~kW})$.

$I_{n i} \quad$ Index of feeder (node) $i(\mathrm{~kW}, \mathrm{~kW})$.

$I_{e k} \quad$ Index of tie-breaker (edge) $k(\mathrm{~kW}, \mathrm{~kW})$.

$C_{i} \quad$ Reserve capacity of feeder $i(k W)$.

$S C_{i} \quad$ Sum of all the reserve capacities of the incident feeders of feeder $i(k W)$.

$R_{i} \quad S C_{i}$ divided by $P_{D i}$.

\section{INTRODUCTION}

$\mathbf{T}$ RADITIONALLY, the reliability of distribution systems is studied from the point of view of system planning and long-term operation and the corresponding performance indices are calculated with the statistic and probabilistic method [1], [2]. However, all of these indices are computed based on historical data and cannot reflect the restorability of power supply (RPS) of distribution systems in coming duration, which is more important for the operators.

The RPS of a distribution system is a new concept. The RPS is the capability of the system to restore the power supply once

Manuscript received June 6, 2002. This work was supported by the National Natural Science Foundation of China under Grant 50007006.

Y. Deng and L. Cai are with the Department of Electrical Engineering, Tsinghua University, Beijing 100084, China (e-mail: dym @tsinghua.edu.cn.).

Y. Ni is with the Department of Electrical and Electronic Engineering, The University of Hong Kong, Hong Kong, China (e-mail: yxni@eee.hku.hk).

Digital Object Identifier 10.1109/TPWRD.2003.817805 a fault or maintenance occurs. It is the measurement of feasibility of restoration schemes and reflects the states of the coming duration.

Since the study of RPS is focused on the feasibility of restoration, in this paper, we suggest applying the service restoration algorithm (i.e., load transfer algorithm), to enhance the RPS of distribution systems.

In the past decades, a lot of algorithms have been presented in the area of service restoration [3]-[12], such as genetic algorithm [3], artificial neural network algorithm [4], heuristic search [5]-[7], a two-stage strategy [8], tabu search [9], fuzzy-GA method [10], etc. All of these papers had done well for the research of service restoration at a certain load level. Miu and Chiang investigated service restoration with varying loads [11]. Rolim, et al. presented an intelligent tool for maintenance scheduling of distribution systems [12].

In this paper, a new heuristic search approach, named feeder incidence diagram (FID), will be developed to improve the restorability of power supply in distribution systems.

Both the research of reliability and restorability should study how to transfer load once faults occur.

There are several ways to study the reliability problem using load transfer

1) Consider the connectivity and neglect the capacity constraint of distribution lines [1].

2) Adopt maximum network flow method to consider the capacity constraint of distribution lines with network loss neglected [2], [13].

3) Adopt Z matrix and dc load flow method for contingency evaluation [14], [15].

A feasible restoration scheme should have sufficient reserve capacities of distribution lines and available network connection relationship. In this paper, based on the service restoration algorithm and the accurate three-phase load flow algorithm, we can obtain feasible schemes very quickly.

\section{Performance Indices of the RPS}

Maximal interrupted power (MIP) and maximal number of interrupted customers (MNIC) can be used as the performance indices of the RPS. MIP and MNIC are the maximal values of interrupted power and customers in a feeder, respectively, after the load transfer in cases of faults or maintenance. MIP can be calculated based on the network topology after fault isolation. Therefore, MIP and MNIC reflect the load transfer capability of a feeder in the worst outage condition [16]. The less the MIP and MNIC are, the better the restorability indices are. 


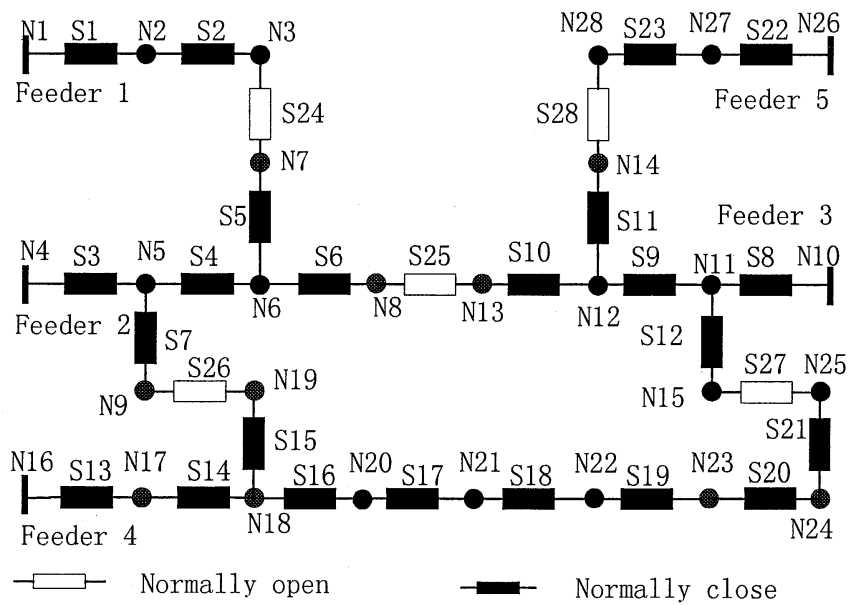

Fig. 1. Illustration of a feeder system.

Considering the load changes in a wide range, the maximum rate of interrupted power is also used

$$
R_{M I P}=\frac{M I P}{P_{D}}
$$

where $R_{M I P}$ is the maximum rate of interrupted power; $P_{D}$ is the total load of the feeder.

There are many ways to enhance the RPS of distribution systems, such as adding tie-breakers and sectionalizers, building new distribution lines, and so on, in which the investment is required. In this paper, we try to improve the RPS via only changing the structure of distribution systems. Network reconfiguration can make systems operate more secure and more economical. Likewise, we can change the network structure via altering the states of open-close pair of circuit breakers and then change the load distribution to enhance the RPS. However, it is extremely time-consuming for validating each possible combination of circuit breakers because of too many combinations. Even in network reconfiguration algorithm [17], it tries to find and validate the best scheme from all of the combinations instead of testing each possible combination.

There are three assumptions, based on which we can see clearly the relationship between the RPS and load transfer and use FID to do the relevant analysis.

a) The load transfer between two feeders will not change the incidence relationships among feeders.

b) Neglect the line loss (i.e., the reserve capacity of a feeder equals to the rated capacity minus total load demand).

c) The RPS of a feeder is directly proportional to the reserve capacities of its incident feeders.

Assumption A is to reduce the combination number of circuit breakers. As seen in Fig. 1, when load is transferred from feeder 2 to feeder 1, we should open circuit breaker S5 instead of S4; otherwise, the incidence relationship between feeder 2 and feeder 3 will be destroyed. Actually, this assumption implies that, in normal case the operations of circuit breakers should not affect the intrinsic structure of the system. The structure of distribution system should be determined by experienced operators.

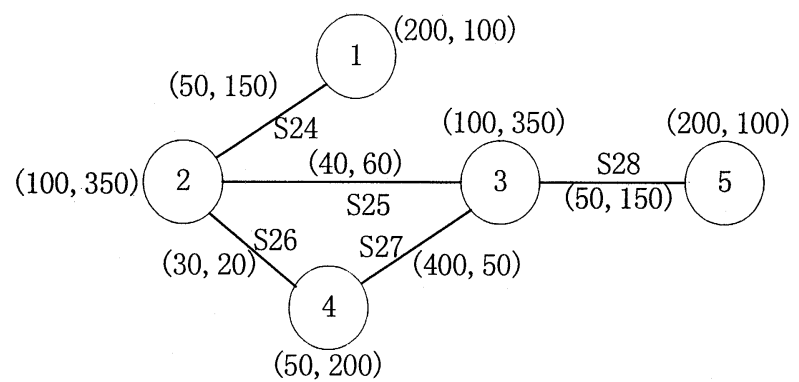

Fig. 2. FID before load transfer (Unit: kilowatts).

The basis of assumption B is that the capacity of a feeder is limited to the rated capacity of the main feeder. In addition, we use the minimum rated capacity of the main feeder as the rated capacity of the feeder.

It is obvious that the performance indices of the RPS, such as MIP, are mainly determined by the interactive back-up capability among feeders. Therefore, according to assumption $\mathrm{C}$, if the reserve capacities of standby feeders are increased, the RPS of this feeder is increased as well. In fact, this assumption neglects the case that the reserve capacity of standby feeder cannot be fully used. The positions of tie-breakers will affect the utilization of the reserve capacities of the relevant feeders. Assumption $\mathrm{C}$ implies that the placement of tie-breaker cabinets has been optimized, and thus, the reserve capacities of feeders can be fully utilized.

\section{FID FOR ANALYZING THE RPS}

Feeders are used as units to calculate the RPS of distribution systems. In addition, the main method to enhance this capability is to transfer load among feeders. If we treat a feeder as a node and the tie-breaker between two feeders as an edge, the feeder incidence diagram (FID) can be built up as seen in Fig. 2 .

The nodes and the edges in Fig. 2 represent the feeders and the tie-breakers in Fig. 1, respectively. The identifiers in Fig. 2, such as S24, S26, etc., are the corresponding tie-breakers. Each node $i$ has an index $I_{n i}$, named feeder index

$$
I_{n i}=\left(C_{i}, S C_{i}\right) \text { for feeder } i
$$

where $C_{i}$ is the reserve capacity of feeder $i$ and $S C_{i}$ is the sum of all the reserve capacities of its incident feeders

$$
S C_{i}=\sum_{j \varepsilon i} C_{j}
$$

The index on edge $k$ named tie-breaker index $I_{e k}$

$$
I_{e k}=\left(P_{t i j}, P_{t j i}\right)
$$

where $i, j$ are the incident nodes of edge $k ; P_{t i j}$ is the load that can be transferred from feeder $i$ to feeder $j$.

\section{A. Illustrations}

In Fig. 2, the index of node 2 is $(100,350)$. It means that the reserve capacity of feeder 2 is $100 \mathrm{~kW}$, the sum of all the reserve capacities of its incident feeders (feeder 1, 3, and 4) is $350 \mathrm{~kW}$ (sum of 200, 100, and $50 \mathrm{~kW}$ ). The index of edge S24 is (50, 150). It means that feeder 2 can transfer $50 \mathrm{~kW}$ to feeder 1 , and feeder 1 can transfer $150 \mathrm{~kW}$ to feeder 2 . 
The RPS of a feeder can be improved by reducing the load of the feeder and increasing the reserve capacities of its incident feeders. Although it is impossible to reduce the total load demand and increase the total reserve capacities of all the feeders through load transfer, the distribution of the reserve capacities of the feeders can be changed via load transfer and the total RPS of the incident feeders can be improved.

In order to effectively improve the distribution of reserve capacities and increase the RPS, we have following targets in load transfer process.

a) After the load transfer, $\sum_{i \in A l l} S C_{i}$ should be increased.

b) After the load transfer, $R_{i}$ of feeder $i$ should not be decreased. $R_{i}$ is defined as

$$
R_{i}=\frac{S C_{i}}{P_{D i}}
$$

where $P_{D i}$ is the total load of feeder $i$. Target $\mathrm{b}$ assures that the reserve capacity or the RPS of a certain feeder will not be weakened by load transfer.

In practical distribution systems, for any feeder, the number of its incident feeders is usually no more than three. With this assumption, the load transfer rules are as follows.

1) In the FID, the load should be transferred from the node with more incident edges to the one with less. If two terminuses of an edge have the same number of incident edges, the load transfer will not occur.

2) The feeder $i$, which has transferred load to another feeder $j$ with only one edge, cannot receive load from other feeders to reduce $C_{i}$. The purpose is to prevent $S C_{j}$ of feeder $j$ from decreasing.

3) When the feeder with two incident edges connects two others with three edges, this feeder should divide its reserve capacity into two parts evenly and accept the load from these two incident feeders. The purpose is to raise $\sum_{i \in A l l} S C_{i}$ and distribute the reserve capacities averagely overall.

We still use Fig. 2 as an illustrative example. It should be noted that $C_{i}$ and $I_{e k}$ are the indices in a period, and thus, we must consider the load variation in the period in our analysis in case of overloading of feeders during load transfer. Therefore, the minimum of $C_{i}$ in the period (i.e., the minimum of the reserve capacity of the feeder will be used). Moreover, the breaker index $I_{e k}$ is set to the minimum of the load transfer as well. According to rule (1), feeder 1 should receive the load from feeder 2 (Close S24 and open S5; transferred capacity is $50 \mathrm{~kW}$ ), and feeder 5 should receive the load from feeder 3 (Close S28 and open S11; transferred capacity is $50 \mathrm{~kW}$ ). According to rule (3), feeder 4 should receive the load from feeder 2 and 3, respectively, (Close S26 and open S7, close S27 and S12; both the transferred capacities are $25 \mathrm{~kW}$ ). In this way, we can get the new FID as shown in Fig. 3. After load transfer, $\sum_{i \in A l l} S C_{i}$ is $1350 \mathrm{~kW}$; it is $250 \mathrm{~kW}$ higher than that before load transfer. The corresponding performance indices are listed in Table I.

From Table I, it can be seen that the load transfer based on the proposed rules can increase not only the reserve capacity of each feeder but also the total reserve capacities of all the five feeders. In addition, the rules can also be used in highly loading cases.

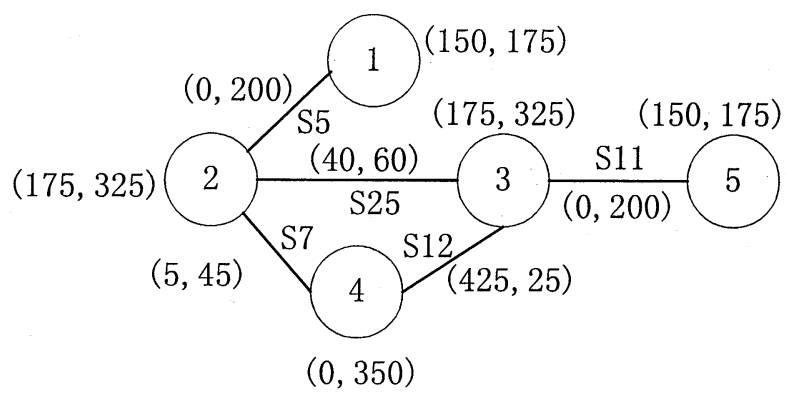

Fig. 3. FID after load transfer (Unit: kilowatts).

TABLE I

Comparison Between the Performance Indices BEFORE AND AFTER LOAD TRANSFER

\begin{tabular}{c|c|c|c|c}
\hline \multirow{2}{*}{$\begin{array}{c}\text { Feeder } \\
\text { Number }\end{array}$} & \multicolumn{2}{|c|}{$P_{D i}(\mathrm{~kW})$} & \multicolumn{2}{c}{$R_{i}$} \\
\cline { 2 - 5 } & $\begin{array}{c}\text { Before } \\
\text { transfer }\end{array}$ & $\begin{array}{c}\text { After } \\
\text { transfer }\end{array}$ & $\begin{array}{c}\text { Before } \\
\text { transfer }\end{array}$ & $\begin{array}{c}\text { After } \\
\text { transfer }\end{array}$ \\
\hline 1 & 150 & 200 & 0.67 & 0.88 \\
\hline 2 & 360 & 285 & 0.97 & 1.14 \\
\hline 3 & 360 & 285 & 0.97 & 1.14 \\
\hline 4 & 500 & 550 & 0.4 & 0.63 \\
\hline 5 & 150 & 200 & 0.67 & 0.88 \\
\hline
\end{tabular}

Through the FID, we can roughly assess the effectiveness of the load transfer. Eventually, we need to calculate the values of MIP and MNIC before and after the load transfer to decide which scheme is better.

\section{Steps of Algorithm IMPlementation}

Step 1: Run the dual topology analysis [18] and power flow [19], and then calculate the performance indices of the RPS before load transfer.

Step 2: Based on the network topology, we can get the corresponding FID. According to the result of power flow calculation, we calculate the reserve capacity and the capacity of the transferable load of each feeder and other performance indices in the FID.

Step 3: According to the load transfer rules, we can develop the scheme of load transfer (i.e., the operated tie-breakers and sectionalizers). Moreover, the performance indices of FID are recalculated. Step 4: According to the load transfer scheme, we carry out the topology analysis and power flow calculation of local area. At the same time, we calculate the performance indices of the RPS after the load transfer. 


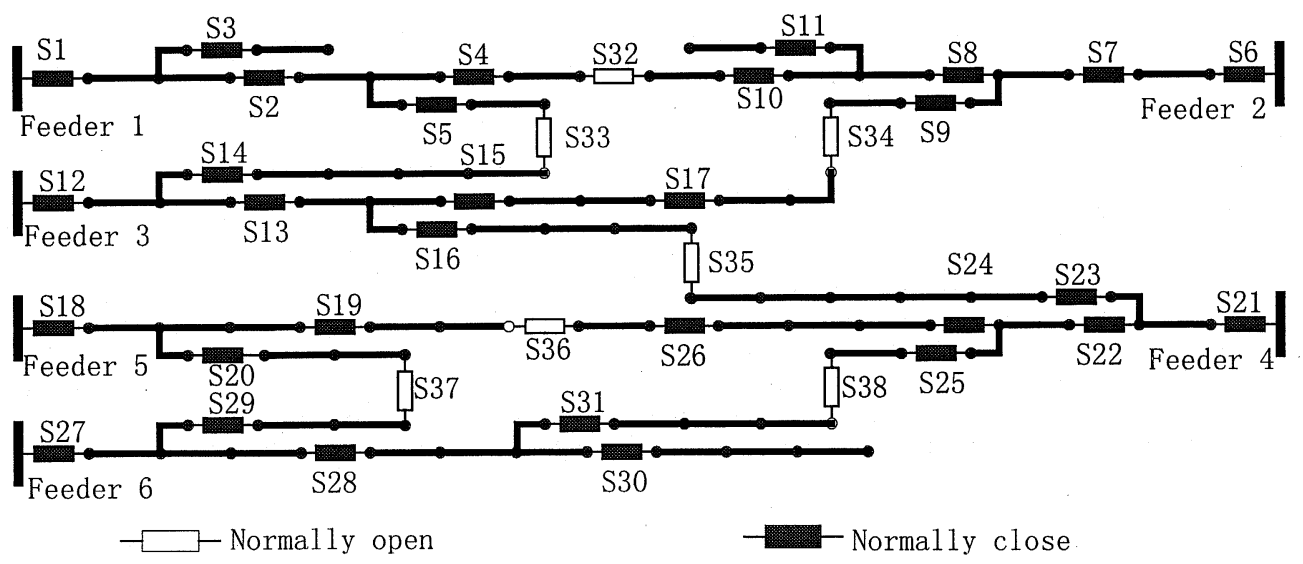

Fig. 4. Distribution system before load transfer.

\section{APPLICATION OF THE RPS INDICES}

\section{A. Reflecting the Operation State of the Feeder}

From the point of view of RPS, the load distribution is very important for the operation state of distribution systems. With the load variation, the RPS of the feeder is also changed. These performance indices can be used as the measurement of the operation state of the feeder.

\section{B. Load Transfer or Switch Operation}

Calculate the corresponding performance indices of the feeder before and after switch operation to determine the influence of the switch operation to the RPS of the feeder.

\section{Optimizing the Placement of Tie-Breakers}

Optimizing the placement of tie-breakers to maximize the RPS of distribution systems is the focus of power supply utilities. Through calculating the MIP of the two feeders, which are the terminuses of the tie-breaker, we can educe the best position of the tie-breaker.

\section{Assessing the Power Restoration Scheme}

Through comparing the MIPs and MNICs of different restoration schemes after restoration calculation, one can decide which restoration scheme is better.

\section{CASE Study}

One may calculate the RPS before and after the load transfer. In Fig. 4, using FID, one can get the load transfer scheme of improving RPS, (i.e., transfer part of the load of feeder 3 to feeder 2 and 1; transfer part of the load of feeder 4 to feeder 5 and 6$)$.

In this load transfer scheme, close tie-breakers S33, S34, S36, and S38, and open breakers S14, S15, S24, and S25 in Fig. 4. Figs. 5 and 6 show the corresponding MIP and MNIC of the system before and after the load transfer, respectively. It is obvious that after the load transfer, the RPS of the whole system is improved. The performance indices of single feeder can also demonstrate this point. Table II shows the change of MIP and

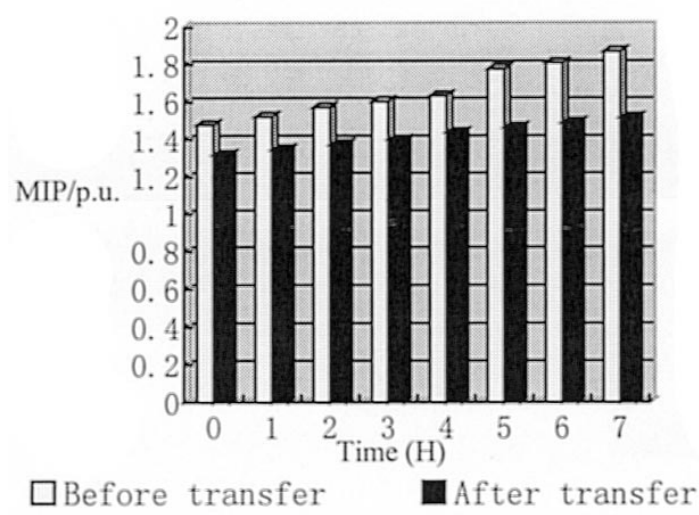

Fig. 5. Comparison between the MIPs before and after load transfer.

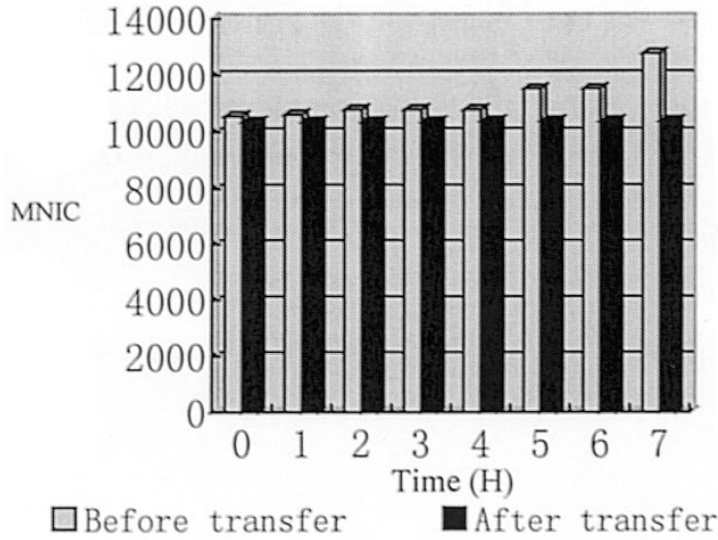

Fig. 6. Comparison between the MNICs before and after load transfer.

MNIC of feeder 3 before and after the load transfer. This change is partly because that feeder 3 transfers a large portion of its load to other feeders.

Table III shows the RPS of feeder 1 before and after the load transfer. After the load transfer, the RPS of feeders is improved. This is because the reserve capacities are concentrated on the feeders with more incident tie-breakers, which makes the RPS of the whole system increase. In addition, for the receiving feeders, although its reserve capacity is decreased by accepting load, the decreasing amount is less than the increasing amount of 
TABLE II

RPS OF FEEDER 3 BEFORE AND AFTER LOAD TRANSFER

\begin{tabular}{c|c|c|c|c}
\hline \multirow{2}{*}{ Index } & \multicolumn{2}{|c|}{ MIP (p.u.) } & \multicolumn{2}{c}{ MNIC } \\
\cline { 2 - 5 } & $\begin{array}{c}\text { Before } \\
\text { transfer }\end{array}$ & $\begin{array}{c}\text { After } \\
\text { transfer }\end{array}$ & $\begin{array}{c}\text { Before } \\
\text { transfer }\end{array}$ & $\begin{array}{c}\text { After } \\
\text { transfer }\end{array}$ \\
\hline 0 & 0 & 0 & 0 & 0 \\
\hline 1 & 0.013 & 0 & 63 & 0 \\
\hline 2 & 0.035 & 0 & 225 & 0 \\
\hline 3 & 0.036 & 0 & 225 & 0 \\
\hline 4 & 0.037 & 0 & 225 & 0 \\
\hline 5 & 0.047 & 0 & 261 & 0 \\
\hline 6 & 0.048 & 0 & 261 & 0 \\
\hline 7 & 0.05 & 0 & 261 & 0 \\
\hline
\end{tabular}

TABLE III

RPS OF FEEDER 1 BEFORE AND AFTER LOAD TRANSFER

\begin{tabular}{c|c|c|c|c}
\hline \multirow{2}{*}{ Index } & \multicolumn{2}{|c|}{ MIP (p.u.) } & \multicolumn{2}{c}{ MNIC } \\
\cline { 2 - 5 } & $\begin{array}{c}\text { Before } \\
\text { transfer }\end{array}$ & $\begin{array}{c}\text { After } \\
\text { transfer }\end{array}$ & $\begin{array}{c}\text { Before } \\
\text { transfer }\end{array}$ & $\begin{array}{c}\text { After } \\
\text { transfer }\end{array}$ \\
\hline 0 & 0.225 & 0.225 & 1448 & 1448 \\
\hline 1 & 0.230 & 0.230 & 1448 & 1448 \\
\hline 2 & 0.234 & 0.234 & 1448 & 1448 \\
\hline 3 & 0.239 & 0.239 & 1448 & 1448 \\
\hline 4 & 0.243 & 0.243 & 1448 & 1448 \\
\hline 5 & 0.349 & 0.248 & 2150 & 1448 \\
\hline 6 & 0.355 & 0.252 & 2150 & 1448 \\
\hline 7 & 0.362 & 0.257 & 2150 & 1448 \\
\hline
\end{tabular}

the reserve capacity of its incident feeders. Therefore, the RPS of those feeders, such as feeder 1, is not weakened.

\section{CONCLUSIONS}

In this paper, a new concept of RPS and the corresponding performance indices are suggested. Feeder incidence diagram (FID) is proposed for analyzing the rules for network structure change decision, and a load transfer algorithm is used for enhancing the RPS of distribution systems. The performance indices, MIP and MNIC, will be calculated before the restoration operation or apparatus maintenance. These indices can serve for evaluating the operation status of feeders, estimating the impact of load transfer and switch operation, and assessing the RPS. They can also be used to optimize the allocation of tie-breakers in a meshed network. The computer simulation results show that the proposed scheme can effectively enhance the RPS of distribution systems.

\section{REFERENCES}

[1] Y. Guo, Principle and Application of Power System Reliability (in Chinese). Beijing, China: Tsinghua Univ. Press, 1986.

[2] R. L. Sullivan, Power System Planning. New York: McGraw-Hill, 1977.

[3] N. G. Bretas, A. C. B. Delbem, and A. de Carvalho, "Optimal energy restoration for general distribution systems by genetic algorithms," in Proc. Int. Conf. Power Syst. Technol., vol. 1, 1998, pp. 43-7.

[4] Y.-Y. Hsu and H.-M. Huang, "Distribution system service restoration using the artificial neural network approach and pattern recognition method," Proc. Inst. Elect. Eng. C - Gen., Transm. Dist., vol. 142, no. 3, pp. 251-6, May 1995.

[5] T. Taylor and D. Lubkeman, "Implementation of heuristic search strategies for distribution feeder reconfiguration," IEEE Trans. Power Delivery, vol. 5, pp. 239-46, Jan. 1990.

[6] K. N. Miu, H.-D. Chiang, B. Yuan, and G. Darling, "Fast service restoration for large-scale distribution systems with priority customers and constraints," IEEE Trans. Power Syst., vol. 13, pp. 789-95, Aug. 1998.

[7] S. Toune, H. Fudo, T. Genji, Y. Fukuyama, and Y. Nakanishi, "Comparative study of modern heuristic algorithms to service restoration in distribution systems," IEEE Trans. Power Delivery, vol. 17, pp. 173-181, Jan. 2002.

[8] R. E. Brown and A. P. Hanson, "Impact of two-stage service restoration on distribution reliability," IEEE Trans. Power Syst., vol. 16, pp. 624-629, Nov. 2001

[9] Y. Fukuyama, "Reactive tabu search for distribution load transfer operation," Proc. IEEE Power Eng. Soc. Winter Meeting, vol. 2, pp. 1301-1306, Jan. 2000.

[10] Y. -T. Hsiao and C. -Y. Chien, "Enhancement of restoration service in distribution systems using a combination fuzzy-GA method," IEEE Trans. Power Syst., vol. 15, pp. 1394-1400, Nov. 2000.

[11] K. N. Miu and H.-D. Chiang, "Service restoration for unbalanced radial distribution systems with varying loads: Solution algorithm," Proc. IEEE Power Eng. Soc. Summer Meeting, vol. 1, pp. 254-258, July 1999.

[12] J. G. Rolim and C. R. da Silva Filho, "An intelligent tool for maintenance scheduling of distribution systems," in Proc. Int. Conf. Elect. Utility Deregulation Restructuring Power Technologies, Apr. 2000, pp. 215-220.

[13] M. E. Khan and J. N. Y. Cheung, "Distribution system reliability evaluation taking circuit capacity into consideration," in Proc. Probabilistic Methods Appl. To Power Syst., Vancouver, BC, 1997, pp. 269-274.

[14] H. E. Brown, "Contingencies evaluated by a Z-matrix method," IEEE Trans. Power Apparat. Syst., vol. PAS-88, pp. 409-412, Apr. 1969.

[15] — - "Interchange capability and contingency evaluation by a Z-matrix method," IEEE Trans. Power App. Syst., vol. PAS-91, pp. 1827-32, Sept./Oct. 1972.

[16] L. Cai, "Study on Reliably Supplying Power in Distribution Systems," MS dissertation (in Chinese), Tsinghua Univ., Dept. Elect. Eng., Beijing, China, 2001.

[17] Y. Deng, "Theory and Algorithm: Distribution Network Optimization," Ph.D. dissertation (in Chinese), Tsinghua Univ., Dept. Elect. Eng., Beijing, China, 1994

[18] Y. He, D. C. Yu, Y. Deng, and J. Lei, "An efficient topology processor for distribution systems," Proc. IEEE Power Eng. Soc. Winter Meeting, vol. 1-3, pp. 824-829, Jan. 2001.

[19] J. Lei, Y. Deng, Y. He, and B. Zhang, "A rigid approach of generalized power flow analysis for distribution systems," Proc. IEEE Power Eng. Soc. Summer Meeting, vol. 1-4, pp. 1047-1052, July 2000.

Youman Deng (SM'00) was born in 1966. He received the B.Eng, M. Eng., and Dr. Eng. degrees all in electrical engineering from Tsinghua University, Beijing, China, in 1989, 1991, and 1995, respectively.

He had been an Associate Professor from 1997 to 2003 and a Principal Investigator from 2000 to 2003 at Tsinghua University, Beijing, China. Currently, he is a Senior Officer of government with the Guangzhou Development District, Guangdong Province, China. 
Le Cai was born in 1977. He received the B.Eng. and M.Eng. degrees in electrical engineering from Tsinghua University, Beijing, China, in 1999 and 2001, respectively.

Currently, he is pursuing the Ph.D. degree at Purdue University, West Lafayette, IN.
Yixin Ni (SM'94) received the B.Eng., M.Eng., and Dr. Eng. degrees in electrical engineering from Tsinghua University, Beijing, China.

Currently, she is with the University of Hong Kong, China. She was a Former Professor and Director of National Power System Lab at Tsinghua University, Beijing, China. Her research interests are power system stability and control, HVDC transmission, FACTS, and power markets. 